\title{
Aprendizagem baseada em equipe: Uma proposta para o ensino de matemática financeira
}

\section{Team-based learning: A proposal for teaching financial mathematics}

\author{
ANDRADE, Allana M. ${ }^{1}$ \\ Da SILVA, Antônio Eduardo M. ${ }^{2}$ \\ SEGATTO, Breno R. ${ }^{3}$
}

\begin{abstract}
Resumo
No presente artigo apresentamos uma proposta de intervenção pedagógica com a metodologia de aprendizagem baseada em equipes (Team-Based Learning - TBL) para o ensino/aprendizagem da disciplina matemática financeira de um curso técnico de administração integrado ao ensino médio. $A$ proposta baseou-se em uma investigação teórica de natureza exploratória com abordagem qualitativa, sendo feita a análise da prática dessa metodologia na literatura. Os resultados encontrados apontam para a eficiência do método do desenvolvimento do trabalho em equipe para uma efetiva aprendizagem significativa.

Palavras-chave: metodologia ativa, aprendizagem baseada em equipes, ensino técnico, matemática financeira.

Abstract

In this article, we present a proposal for a pedagogical intervention with the team-based learning methodology (Team-Based Learning - TBL) for the teaching/learning of the financial mathematics discipline of a technical administration course integrated to high school. The proposal was based on a theoretical investigation of an exploratory nature with a qualitative approach, analyzing the practice of this methodology in the literature. The results found point to the efficiency of the teamwork development method for an effective meaningful learning.

Keywords: active methodology, team-based learning, technical course, financial mathematics.
\end{abstract}

\section{Introdução}

Em todos os níveis de ensino encontramos práticas pedagógicas tradicionais cujos métodos de ensino fundamentam-se em aula expositiva, dando-se ênfase à repetição e memorização dos conteúdos e predominando a postura passiva do aluno, bem como a centralização do conteúdo no professor. Isoladamente, a metodologia tradicional que coloca o aluno como passivo no processo de aprendizagem, não atende mais as necessidades do estudante da chamada geração digital, fazendo com que o professor se adapte e busque novas ações pedagógicas compatíveis com as demandas da sociedade.

${ }^{1}$ Instituto Federal do Espírito Santo, campus Colatina-ES. Brasil. allana@ifes.edu.br.

2Instituto Federal do Espírito Santo, campus Colatina-ES. Brasil. antonio.eduardo@ifes.edu.br

${ }^{3}$ Universidade Federal do Espirito Santo, Programa de Pós-Graduação em Ensino de Física, Viória-ES. Brasil. breno.segatto@ufes.br 
Para superar esse desafio, as práticas pedagógicas diferenciadas, com metodologias ativas de ensino tem crescido no ambiente educacional em diferentes áreas do conhecimento. Essas rompem com o método tradicional de ensino e baseiam-se em uma pedagogia problematizadora que estimula o aluno a adotar uma postura ativa, buscando a autonomia e a aprendizagem significativa (Paiva et al., 2016).

No ensino da matemática financeira, a metodologia tradicional com aulas expositivas centradas no professor, ainda é muito frequente. No entanto, estratégias que motivem o aluno a uma participação ativa promovem a aprendizagem significativa, além de desenvolver o senso crítico (Pinto et al., 2012). Dessa forma, essa pesquisa tem a seguinte questão de investigação: Como melhorar o processo de ensino-aprendizagem de Matemática Financeira no curso técnico de administração integrado ao ensino médio de forma a desenvolver competências que contribuam para formar profissionais ativos, críticos e reflexivos?

\section{Referencial teórico}

Neste capítulo é apresentado o referencial teórico que embasou a proposição da intervenção pedagógica. Abordam-se os principais conceitos, definições e etapas do método de aprendizagem baseada em equipe, além do conteúdo específico de séries uniformes de pagamentos trabalhado na intervenção pedagógica.

\subsection{Aprendizagem baseada em equipe}

A aprendizagem baseada em equipe, do inglês Team-Based Learning (TBL), é uma metodologia criada nos anos 70 pelo professor da área de negócios Larry K. Michaelsen na Universidade de Oklahoma (EUA) com o intuito de desenvolver o estudo colaborativo de forma contextualizada. O método TBL tem como foco melhorar a aprendizagem a partir de um conjunto de tarefas e atividades que coloca o aluno como responsável por adquirir conhecimentos, além de proporcionar o desenvolvimento de várias competências como tomada de decisão, trabalho colaborativo em equipe, raciocínio crítico, entre outras (Krug et al., 2016).

O desenvolvimento da aprendizagem baseada em equipe oportuniza o estudante adquirir o conhecimento da disciplina por meio de uma sequência de atividades. Cada disciplina é estruturada em módulos e cada módulo é desmembrado em atividades de preparação e aplicação dentro e fora da sala de aula (T. E. de Oliveira et al., 2016).

Segundo Krug et al. (2016), o módulo requer três etapas de atividades: o preparo, a garantia do preparo e a aplicação dos conceitos. O preparo envolve o estudo prévio do aluno, fora da sala de aula, de acordo com as tarefas propostas pelo professor. A garantia do preparo consiste na aplicação, em sala de aula, de um teste individual que será posteriormente aplicado em equipe com feedback, possibilidade de questionamento, caso haja discordância da resposta, e breve apresentação do professor. Na aplicação dos conceitos executa-se atividades em equipe para tomada de decisões e resoluções dos problemas propostos pelo professor, com apresentação e feedback ao final.

As atividades de preparação e aplicação dos conceitos, no método TBL, são ilustradas por T. E. de Oliveira (2016), conforme Figura 1, em quatro etapas. A primeira etapa destaca a fase de preparação da aprendizagem com a realização de estudo prévio pelos alunos a partir da leitura do conteúdo, vídeos, simulações, etc. que deverão ser disponibilizados antecipadamente aos alunos. Já a etapa 2 aborda a verificação da aprendizagem da fase de preparação com a realização de um teste de preparação individual (TPi) e logo após, o teste de preparação em equipe (TPe), seguido por apelação e breve exposição do professor sobre os pontos de maior dificuldade. Na etapa 3, ocorre as tarefas de aplicação extraclasse com complexidade gradual. Já em sala de aula, as atividades do tipo resolução de problemas, com contextualização, são discutidas em equipe para solução, conforme demonstrado na etapa 4 da Figura 1. 
Figura 1

Principais fases de cada módulo do TBL

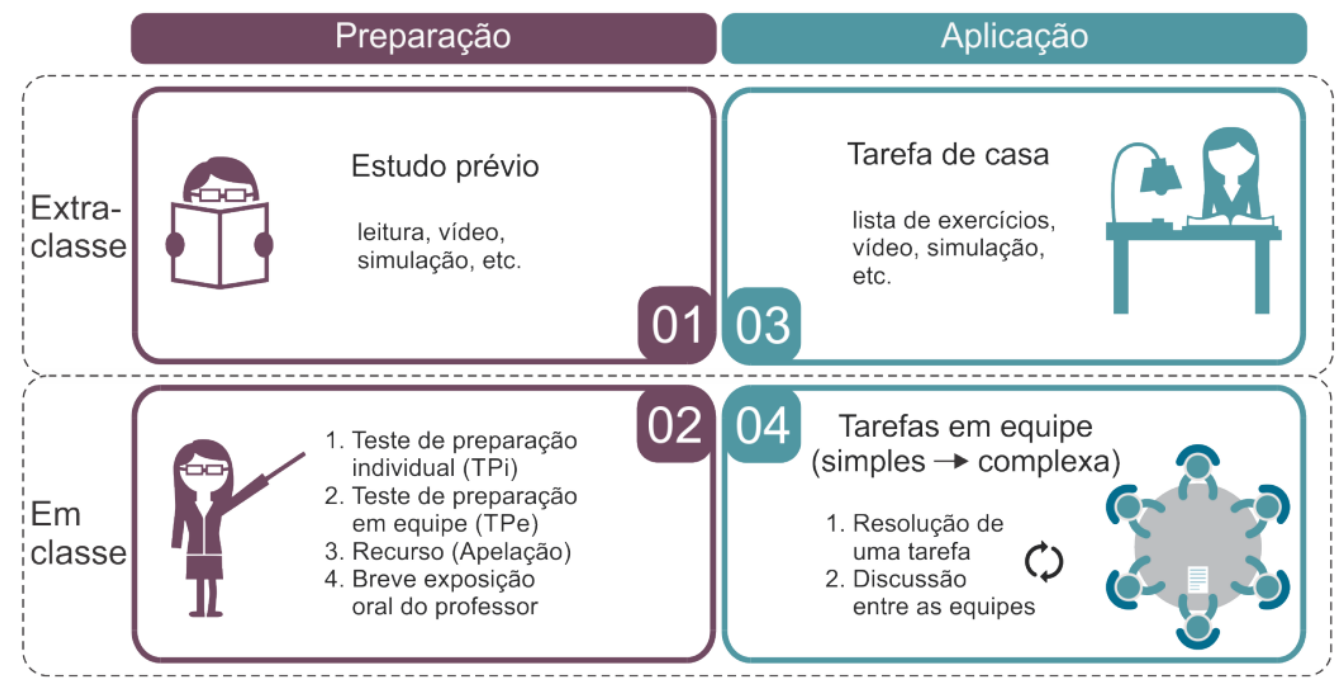

Fonte: (T. E. de Oliveira, 2016)

A aprendizagem baseada em equipe surge como uma metodologia ativa alternativa a métodos tradicionais de ensino, colocando o professor como mediador na construção do conhecimento do aluno e considerando as experiências e conhecimentos prévios dos estudantes na busca de uma aprendizagem significativa. Para essa construção de significados Pinto et al. (2012) afirma que o aluno deve ser o protagonista de sua aprendizagem, desenvolvendo o senso crítico e a capacidade de relacionar os conteúdos teóricos a contextos reais.

\section{Metodologia}

Com o intuito de atender ao objetivo geral da pesquisa que é apresentar uma proposta de intervenção pedagógica com a metodologia de aprendizagem baseada em equipes (TBL) para o ensino/aprendizagem da disciplina matemática financeira do curso técnico de administração integrado ao ensino médio, esse estudo se estabelece como uma pesquisa teórica do tipo exploratória com abordagem qualitativa, visto que é uma forma de explorar o significado de um problema, sendo fundamentalmente interpretativa (Richardson, 1999).

Por ser um tema pouco abordado, especialmente no nível técnico de ensino, a pesquisa exploratória se mostra adequada, pois o que se espera é apresentar uma estratégia de ensino tornando o método TBL mais explícito (Gil, 2002).

Em virtude da pandemia do COVID-19, a intervenção pedagógica proposta não foi aplicada. No entanto, com base nos procedimentos técnicos, sua aplicação se caracteriza como uma pesquisa-ação, já que o pesquisador (professor) e participantes (alunos) estão envolvidos no processo de pesquisa (Gil, 2002) e a aplicação do método TBL com o conteúdo de séries uniformes de pagamentos da disciplina matemática financeira deve ser realizada pelo próprio professor.

A estratégia educacional proposta tem como público-alvo os alunos do segundo ano do curso técnico em administração integrado ao ensino médio do Instituto Federal do Espírito Santo - campus Colatina, o que corresponde a uma amostra de 40 alunos aproximadamente, divididos em 8 grupos com 5 integrantes, com idades entre 15 e 18 anos. 
A disciplina de matemática financeira possui uma carga horária total de 60h no curso técnico de administração integrado ao ensino médio e a proposta de intervenção pedagógica com o conteúdo de séries uniformes de pagamentos corresponderá a uma carga horária de $15 \mathrm{~h}$.

A pesquisa proposta na prática pedagógica é de corte transversal, e, a princípio, terá dados selecionados em um período de vinte e dois dias, utilizando técnicas de coleta de dados como observação e questionário (Gil, 2002). Na observação, o pesquisador examina fatos ou fenômenos que orientam as atitudes comportamentais dos indivíduos (Marconi \& Lakatos, 2010), sendo aplicado nessa pesquisa com o intuito de observar a participação, a motivação e as impressões favoráveis ou desfavoráveis dos alunos ao realizar a prática pedagógica proposta.

O questionário de Masocatto et al., (2019) será enviado para aprovação do comitê de ética em pesquisa (CEP/Ifes) e disponibilizado por meio da plataforma Google Forms, juntamente com o Termo de Consentimento Livre e Esclarecido (TCLE), com o intuito de analisar a percepção dos estudantes quanto à contribuição da prática pedagógica na construção do seu conhecimento. Os dados coletados no questionário serão tabulados no Excel e avaliados por meio de análise descritiva a partir dos percentuais das respostas das variáveis.

\subsection{Revisão de literatura}

Na pesquisa realizada no Google Acadêmico e por acesso remoto ao conteúdo assinado do portal de periódicos Capes através da comunidade acadêmica federada - CAFe, disponível para o Instituto Federal do Espírito Santo, observou-se que o método TBL tem sido bastante empregado como estratégia educacional no ensino superior da área de saúde em instituições brasileiras, como os estudos realizados por Anjos, R., Rodriguez, J., Fujiki, R., \& Bernardo, (2016), Barbiero et al., (2017), Krug et al., (2016), Masocatto et al., (2019), Vilela et al., (2017), entre outros, e em instituições estrangeiras nos cursos da área de negócios (Saldivar, 2015; Huang \& Lin, 2017; Smart et al., 2015) e de saúde (Zgheib et al., 2016; Kibble et al., 2016). Os descritores utilizados na busca foram "aprendizagem baseada em equipe" e "team-basedlearning" com recorte temporal de 2015 a 2020.

Os resultados encontrados nos estudos da área de saúde indicam que a aprendizagem baseada em equipe é um excelente método pedagógico para a aprendizagem dos estudantes. Masocatto et al., (p. 110, 2019) afirmam que "uma das vantagens foi a capacidade de desenvolver habilidades em raciocínio clínico e a possibilidade de construir o conhecimento com sua utilização prática". Já Krug et al., (2016) destacam ainda outras competências com a aplicação do método TBL, como o trabalho colaborativo e efetivo em equipe e a tomada de decisão. Entretanto, Bollela et al., (2014) alertam que para se ter um eficiente desempenho, os grupos precisam ser heterogêneos, cada aluno deve ser responsabilizado pelas atividades individuais e em equipe, as tarefas devem promover o desenvolvimento e a aprendizagem do grupo e os alunos precisam receber feedback frequentemente durante a aplicação do método.

Apesar de amplamente empregada nos cursos superiores da área de saúde, a metodologia de aprendizagem baseada em equipe foi aplicada nos estudos de T. E. de Oliveira et al., (2016), como estratégia de ensino no curso superior de física e também demonstrou resultados positivos nesta área. Os resultados encontrados apontam que "a resolução de questões conceituais e problemas em sala de aula e a interação prolongada com os membros da equipe, atuaram como potenciais fontes geradoras de autoeficácia em aprender física e em trabalhar colaborativamente" (T. E. de Oliveira et al., 2016, p. 977).

O método TBL também foi aplicado no curso superior de administração no estudo de Camargo et al., (2017), como estratégia de avaliação formativa. Os resultados do estudo sugerem que o método TBL coloca o aluno como responsável pela pontuação obtida individualmente e em grupo e protagonista no processo ensinoaprendizagem, o que contribui significativamente para o desenvolvimento profissional e acadêmico (Camargo et al., 2017). 
No nível médio, o método TBL foi aplicado no curso técnico de administração por Giacomelli, (2020), na disciplina de processos operacionais contábeis. Giacomelli, (2020) destaca que os resultados alcançados foram superiores aos de outros métodos e que o índice de acertos das repostas individuais para as respostas em equipe, foi crescente. A autora ressaltou ainda que o método proporcionou a aprendizagem significativa do conteúdo e a participação ativa dos alunos.

Quando os descritores da pesquisa no portal de periódicos Capes utilizados foram "team-basedlearning" and "matemática financeira", sem recorte temporal, encontrou-se zero resultados. Contudo, no Google Acadêmico, encontrou-se o estudo de Uzinski et al., (2019) que apresenta reflexões sobre o ensino-aprendizagem de matemática financeira nos cursos superiores de ciências contábeis e licenciatura em matemática, com aplicação de diferentes metodologias, entre elas a aprendizagem baseada em equipe, sendo também atestado o aumento no desempenho dos alunos com o uso desse método.

A percepção dos estudantes quanto à aplicação da metodologia de aprendizagem baseada em equipe também é positiva. Os estudos de Barbiero et al., (2017) e Masocatto et al., (2019) demonstram que os estudantes se sentiram mais motivados a estudar o conteúdo e aprovaram a utilização do método. "A utilização do método foi percebida como favorável nos aspectos de preferência (em relação à aula expositiva tradicional), motivação, satisfação e aprendizado" (Masocatto et al., p. 110, 2019).

Os estudos, decorrentes da aplicação de práticas pedagógicas com a aprendizagem baseada em equipe (TBL), adotam processos metodológicos semelhantes. Geralmente, trata-se de uma pesquisa quali-quantitativa com coleta de dados através de observação e aplicação de questionários, além de entrevista em alguns casos. Para a análise dos dados, muitos estudos analisam a estatística descritiva e/ou a frequência absoluta/relativa dos dados.

Dentre os desafios encontrados na aplicação do método, estão a resistência de alguns alunos, do setor administrativo da instituição de ensino e dos próprios colegas docentes, principalmente no início da aplicação do método ( T. E de OLIVEIRA et al., 2016). Por isso, apresentar inicialmente a metodologia de aprendizagem baseada em equipe, bem como mostrar os objetivos educacionais e como eles são alcançados no método, é fundamental para o êxito da prática pedagógica.

\subsection{Proposta de intervenção pedagógica}

A intervenção pedagógica proposta nesta pesquisa visa proporcionar o conhecimento e contextualização dos conteúdos teóricos de séries uniformes de pagamentos da disciplina Matemática Financeira mediante aplicação da metodologia baseada em equipes (TBL) no curso técnico de administração integrado ao ensino médio. A proposta coloca o aluno como protagonista no processo de ensino-aprendizagem e estimula o trabalho colaborativo e efetivo em equipe, desenvolvendo atividades de preparação e aplicação em classe e extraclasse, conforme planejamento sintetizado no Quadro 2.

Antes de iniciar a aplicação da prática pedagógica, é fundamental explicar todo o processo de ensinoaprendizagem do método TBL, bem como as formas de avaliação. Mostrar os objetivos educacionais e explicar como eles são alcançados no método é essencial para o êxito da prática pedagógica. 
Quadro 2

Planejamento da atividade pedagógica

\begin{tabular}{|c|c|c|c|c|}
\hline Atividade & Data & Descrição & $\begin{array}{c}\mathrm{CH} \\
\text { Presencial }\end{array}$ & $\begin{array}{l}\mathrm{CH} \\
\mathrm{EaD}\end{array}$ \\
\hline Apresentação & $\begin{array}{l}\text { Aula Presencial } \\
\text { Data a ser definida }\end{array}$ & $\begin{array}{c}\text { Apresentação do método TBL, da prática } \\
\text { pedagógica e do Ambiente Virtual de } \\
\text { Aprendizagem (Moodle), além da divisão das } \\
\text { equipes. }\end{array}$ & $1 \mathrm{~h} 40$ & - \\
\hline Etapa 1 & $\begin{array}{l}\text { Aula Não Presencial } \\
\text { Data a ser definida }\end{array}$ & $\begin{array}{l}\text { Preparação extraclasse: estudo prévio do } \\
\text { conteúdo no AVA - Moodle. }\end{array}$ & - & $6 \mathrm{~h}$ \\
\hline Etapa 2 & $\begin{array}{l}\text { Aula Presencial } \\
\text { Data a ser definida }\end{array}$ & $\begin{array}{c}\text { Garantia do preparo: aplicação do teste } \\
\text { individual e em equipe, apelação e feedback } \\
\text { do professor. Jogo de tabuleiro. }\end{array}$ & $2 \mathrm{~h} 40$ & \\
\hline Etapa 3 & $\begin{array}{l}\text { Aula Não Presencial } \\
\text { Data a ser definida }\end{array}$ & $\begin{array}{l}\text { Aplicação extraclasse: resolução de } \\
\text { simulações práticas do conteúdo. }\end{array}$ & - & $3 h$ \\
\hline Etapa 4 & $\begin{array}{c}\text { Aula Presencial } \\
\text { Data a ser definida }\end{array}$ & $\begin{array}{c}\text { Aplicação: resolução em equipe de situações } \\
\text { práticas. }\end{array}$ & $1 \mathrm{~h} 40$ & \\
\hline \multicolumn{3}{|c|}{ Carga Horária Presencial } & \multicolumn{2}{|l|}{$6 \mathrm{~h}$} \\
\hline \multicolumn{3}{|c|}{ Carga Horária EaD } & \multicolumn{2}{|l|}{$9 \mathrm{~h}$} \\
\hline \multicolumn{3}{|c|}{ Carga Horária Total } & \multicolumn{2}{|l|}{$15 \mathrm{~h}$} \\
\hline
\end{tabular}

Fonte: Os autores (2020).

A formação das equipes também é realizada nesse primeiro momento presencial que está descrito no Quadro 3. Os grupos, com 5 alunos, são formados pelo professor, mesclando diferentes características dos estudantes e garantindo a diversidade em cada equipe.

Quadro 3

Plano de aula da apresentação

\begin{tabular}{|c|c|c|c|c|c|c|}
\hline \multicolumn{2}{|c|}{ Data } & \multicolumn{4}{|c|}{ A ser definida } & \\
\hline \multicolumn{2}{|c|}{ Tema } & \multicolumn{4}{|c|}{ Apresentação da prática pedagógica. } & \\
\hline \multicolumn{2}{|c|}{ Objetivos } & \multicolumn{4}{|c|}{$\begin{array}{l}\text { Conhecer o método TBL. } \\
\text { so de ensino-aprendizagem da prática pedagógica. } \\
\text { o Ambiente Virtual de Aprendizagem. } \\
\text { Dividir os alunos em grupos. }\end{array}$} & \\
\hline \multicolumn{2}{|c|}{ Conteúdos } & \multicolumn{4}{|c|}{ Aprendizagem baseada em equipe } & \\
\hline \multicolumn{2}{|c|}{$\begin{array}{l}\text { Unidade } \\
\text { Didática }\end{array}$} & Metodologia & $\begin{array}{l}\text { Recursos } \\
\text { Didáticos }\end{array}$ & $\begin{array}{c}\text { Tipo de } \\
\text { Atividade }\end{array}$ & Avaliação & Pontos \\
\hline 1 & $\mathrm{On}$ & $\begin{array}{l}\text { Aula expositiva } \\
\text { dialogada. Vídeo } \\
\text { demonstrativo. }\end{array}$ & $\begin{array}{l}\text { Quadro. Pincel. } \\
\text { Computador. } \\
\text { Data show. Caixa } \\
\text { de som }\end{array}$ & Presencial & $\begin{array}{l}\text { Avaliação } \\
\text { diagnóstica }\end{array}$ & - \\
\hline 2 & $\begin{array}{l}\text { Amb } \\
\text { de } A p\end{array}$ & $\begin{array}{c}\text { Aula expositiva } \\
\text { dialogada. Objeto de } \\
\text { aprendizagem }\end{array}$ & $\begin{array}{l}\text { Quadro. Pincel. } \\
\text { Computadores. } \\
\text { AVA Moodle. }\end{array}$ & Presencial & $\begin{array}{l}\text { Avaliação } \\
\text { diagnóstica }\end{array}$ & - \\
\hline 3 & & $\begin{array}{l}\text { Distribuir os alunos em } \\
\text { grupos }\end{array}$ & Quadro. Pincel. & Presencial & $\begin{array}{c}\text { Avaliação } \\
\text { diagnóstica }\end{array}$ & - \\
\hline
\end{tabular}

Fonte: Os autores (2020).

A proposta de intervenção pedagógica inicia-se com a preparação extraclasse. Todo o conteúdo teórico de séries uniformes de pagamentos é disponibilizado antecipadamente no em uma plataforma online (AVA-Moodle) com 
indicação de leitura, vídeos e exercícios que devem ser resolvidos com o uso das funções financeiras de planilhas eletrônicas, conforme descrito no Quadro 4. Os exercícios são disponibilizados como uma atividade questionário no Moodle, proporcionando uma avaliação diagnóstica e formativa no valor de 1,0 ponto. Esta etapa da proposta pedagógica afeta diretamente o desempenho individual e, como consequência, o da equipe, já que coloca o aluno como protagonista na construção do conhecimento.

\section{Quadro 4}

Plano de aula Etapa 1

\begin{tabular}{|c|c|c|c|c|c|c|c|}
\hline \multicolumn{2}{|c|}{ Data } & \multicolumn{6}{|c|}{ A ser definida } \\
\hline \multicolumn{2}{|c|}{ Tema } & \multicolumn{6}{|c|}{ Séries Uniformes de Pagamentos. } \\
\hline \multicolumn{2}{|c|}{ Objetivos } & \multicolumn{6}{|c|}{$\begin{array}{l}\text { Compreender o conceito de séries uniformes de pagamentos. } \\
\qquad \begin{array}{l}\text { Realizar as leituras indicadas. } \\
\text { Assistir aos vídeos propostos. } \\
\text { Resolver os exercícios propostos. }\end{array}\end{array}$} \\
\hline \multicolumn{2}{|c|}{ Conteúdos } & \multicolumn{6}{|c|}{ Séries postecipadas, antecipadas, diferidas e perpétuas; Funções financeiras em planilhas eletrônicas } \\
\hline \multicolumn{3}{|c|}{$\begin{array}{l}\text { Unidade } \\
\text { Didática }\end{array}$} & Metodologia & $\begin{array}{l}\text { Recursos } \\
\text { Didáticos }\end{array}$ & $\begin{array}{c}\text { Tipo de } \\
\text { Atividade }\end{array}$ & Avaliação & Pontos \\
\hline 1 & \multicolumn{2}{|c|}{$\begin{array}{l}\text { Séries uniformes } \\
\text { de pagamentos }\end{array}$} & Leitura e vídeo. & AVA Moodle & $\mathrm{EaD}$ & $\begin{array}{c}\text { Avaliação } \\
\text { diagnóstica }\end{array}$ & - \\
\hline 2 & \multicolumn{2}{|c|}{$\begin{array}{l}\text { Planilhas } \\
\text { eletrônicas }\end{array}$} & Leitura e vídeo & AVA Moodle & $\mathrm{EaD}$ & $\begin{array}{c}\text { Avaliação } \\
\text { diagnóstica }\end{array}$ & - \\
\hline 3 & \multicolumn{2}{|c|}{ Questionário } & $\begin{array}{l}\text { Questões objetivas de } \\
\text { múltipla escolha sobre o } \\
\text { conteúdo }\end{array}$ & AVA Moodle & $\mathrm{EaD}$ & $\begin{array}{c}\text { Avaliação } \\
\text { diagnóstica e } \\
\text { formativa }\end{array}$ & 1,0 \\
\hline \multicolumn{8}{|c|}{ Referências: Assaf Neto, (2019); Brano (2015). } \\
\hline
\end{tabular}

Fonte: Os autores (2020).

$\mathrm{Na}$ etapa 2 da proposta de intervenção pedagógica, a garantia do preparo é verificada presencialmente, em sala de aula, com a aplicação de um teste individual sem consulta, com duração de 30 minutos, disponibilizado como uma atividade questionário no Moodle, proporcionando uma avaliação formativa no valor de 2,0 pontos. Em seguida, os grupos se reúnem para uma discussão colaborativa e resolução do mesmo teste, porém, agora, em equipe, que tem feedback imediato e duração de 50 minutos. Se houver apelação, dúvidas ou questionamentos, o professor utilizará 20 minutos para os esclarecimentos a fim de solidificar a compreensão do conteúdo.

Como forma de consolidar o conteúdo abordado de forma lúdica e atrativa, é proposto um jogo de tabuleiro de perguntas e respostas entre as equipes com pontuações para avançar nas casas do tabuleiro se a resposta estiver correta, e recuar caso contrário, vencendo a equipe que chegar primeiro ao final. Ao jogar o dado e parar em uma casa com interrogação, o aluno da rodada, juntamente com sua equipe, terá até 2 minutos para responder à pergunta e verificar se vai avançar ou recuar nas casas do tabuleiro. As perguntas do jogo serão disponibilizadas como uma atividade questionário no Moodle, que randomizará 50 questões numéricas abertas e terá no feedback da resposta a orientação para o jogador mover o seu peão no tabuleiro de acordo com o resultado certo ou errado.

O planejamento da etapa 2 é apresentado no Quadro 5. 
Quadro 5

Plano de aula Etapa 2

\begin{tabular}{|c|c|c|c|c|c|c|c|}
\hline \multicolumn{2}{|c|}{ Data } & \multicolumn{6}{|c|}{ A ser definida } \\
\hline \multicolumn{2}{|c|}{ Tema } & \multicolumn{6}{|c|}{ Séries Uniformes de Pagamentos. } \\
\hline \multicolumn{2}{|c|}{ Objetivos } & \multicolumn{6}{|c|}{$\begin{array}{l}\text { Compreender o conceito de séries uniformes de pagamentos. } \\
\text { Verificar a aprendizagem do conteúdo. } \\
\text { Consolidar os conceitos de forma lúdica }\end{array}$} \\
\hline \multicolumn{2}{|c|}{ Conteúdos } & \multicolumn{6}{|c|}{ Séries postecipadas, antecipadas, diferidas e perpétuas; Funções financeiras em planilhas eletrônicas. } \\
\hline \multicolumn{3}{|c|}{$\begin{array}{l}\text { Unidade } \\
\text { Didática }\end{array}$} & Metodologia & $\begin{array}{l}\text { Recursos } \\
\text { Didáticos }\end{array}$ & $\begin{array}{c}\text { Tipo de } \\
\text { Atividade }\end{array}$ & Avaliação & Pontos \\
\hline 1 & \multicolumn{2}{|c|}{ Teste individual } & $\begin{array}{l}\text { Questões objetivas de } \\
\text { múltipla escolha sobre o } \\
\text { conteúdo. }\end{array}$ & $\begin{array}{l}\text { Planilha } \\
\text { eletrônica. } \\
\text { AVA Moodle }\end{array}$ & Presencial & $\begin{array}{l}\text { Avaliação } \\
\text { formativa }\end{array}$ & 1,5 \\
\hline 2 & \multicolumn{2}{|c|}{ Teste em equipe } & $\begin{array}{l}\text { Questões objetivas de } \\
\text { múltipla escolha sobre o } \\
\text { conteúdo. }\end{array}$ & $\begin{array}{l}\text { Planilha } \\
\text { eletrônica. } \\
\text { AVA Moodle. }\end{array}$ & Presencial & $\begin{array}{l}\text { Avaliação } \\
\text { formativa }\end{array}$ & 1,5 \\
\hline 3 & \multicolumn{2}{|c|}{ Feedback } & $\begin{array}{c}\text { Aula expositiva } \\
\text { dialogada }\end{array}$ & $\begin{array}{l}\text { Computador. } \\
\text { Data show. }\end{array}$ & Presencial & $\begin{array}{l}\text { Avaliação } \\
\text { formativa }\end{array}$ & - \\
\hline 4 & \multicolumn{2}{|c|}{ Ludicidade } & $\begin{array}{l}\text { Atividade em grupo: } \\
\text { jogo de tabuleiro }\end{array}$ & $\begin{array}{l}\text { Tabuleiro. Dado. } \\
\text { Peão. AVA- } \\
\text { Moodle. }\end{array}$ & Presencial & $\begin{array}{l}\text { Avaliação } \\
\text { formativa }\end{array}$ & - \\
\hline \multicolumn{8}{|c|}{ Referências: Assaf Neto, (2019); Brano (2015). } \\
\hline
\end{tabular}

Fonte: Os autores (2020)

Após a fase de preparação, inicia-se a fase de aplicação dos conceitos. Na etapa 3 , os alunos resolvem uma atividade contextualizada, individualmente, extraclasse e assistem ao vídeo com aplicações do conteúdo de séries uniformes de pagamentos, além de construírem um mural interativo colaborativo, em grupo, com notícias, vídeos, exemplos práticos, entre outros, utilizando a ferramenta online Padlet, a fim de promover uma aprendizagem significativa. A questão de aplicação contextualizada é disponibilizada como uma atividade tarefa no Moodle, conforme exposto no Quadro 6.

Quadro 6

Plano de aula Etapa 3

\begin{tabular}{|c|c|c|c|c|c|c|c|}
\hline \multicolumn{2}{|c|}{ Data } & \multicolumn{6}{|c|}{ A ser definida } \\
\hline \multicolumn{2}{|c|}{ Tema } & \multicolumn{6}{|c|}{ Séries Uniformes de Pagamentos. } \\
\hline \multicolumn{2}{|c|}{ Objetivos } & \multicolumn{6}{|c|}{$\begin{array}{l}\text { Identificar a aplicação de séries uniformes de pagamentos em situações do cotidiano. } \\
\text { Resolver atividade contextualizada. }\end{array}$} \\
\hline \multicolumn{2}{|c|}{ Conteúdos } & \multicolumn{6}{|c|}{ Séries postecipadas, antecipadas, diferidas e perpétuas; Funções financeiras em planilhas eletrônicas. } \\
\hline \multicolumn{3}{|c|}{$\begin{array}{l}\text { Unidade } \\
\text { Didática }\end{array}$} & Metodologia & $\begin{array}{l}\text { Recursos } \\
\text { Didáticos }\end{array}$ & $\begin{array}{l}\text { Tipo de } \\
\text { Atividade }\end{array}$ & Avaliação & Pontos \\
\hline 1 & \multicolumn{2}{|c|}{$\begin{array}{l}\text { Aplicação dos } \\
\text { conceitos }\end{array}$} & Vídeo & AVA Moodle & EaD & $\begin{array}{l}\text { Avaliação } \\
\text { diagnóstica }\end{array}$ & - \\
\hline 2 & \multicolumn{2}{|c|}{ Tarefa } & $\begin{array}{l}\text { Atividade individual: } \\
\text { aplicação } \\
\text { contextualizada }\end{array}$ & AVA Moodle & EaD & $\begin{array}{l}\text { Avaliação } \\
\text { formativa }\end{array}$ & 1,0 \\
\hline 3 & \multicolumn{2}{|c|}{ Contextualização } & $\begin{array}{l}\text { Atividade em grupo: } \\
\text { mural interativo }\end{array}$ & Padlet & EaD & $\begin{array}{l}\text { Avaliação } \\
\text { formativa }\end{array}$ & 1,0 \\
\hline \multicolumn{8}{|c|}{ Referências: Assaf Neto, (2019); Brano (2015). } \\
\hline
\end{tabular}

Fonte: Os autores (2020) 
Na última etapa da proposta pedagógica com o método TBL, é realizada a aplicação dos conceitos, em equipe, na sala de aula. Os grupos se reúnem para discussão e resolução de uma situação prática, mais complexa que a da etapa anterior, que promova a troca de opiniões entre os integrantes da equipe. A situação-problema para resolução simula contextos reais e possui características e complexidade semelhantes às dificuldades encontradas por um técnico em Administração na rotina de suas atividades profissionais, devendo ser resolvida com o uso de planilha eletrônica, conforme demonstrado no Quadro 7.

Quadro 7

Plano de aula Etapa 4

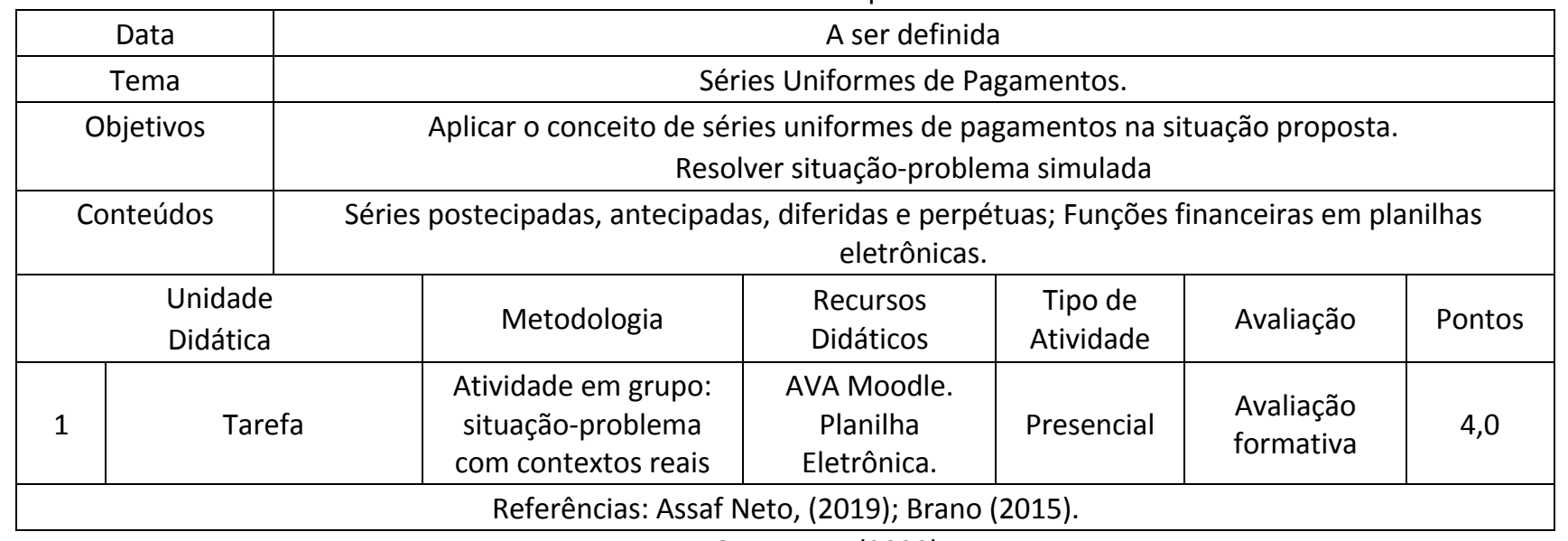

Fonte: Os autores (2020).

Os mecanismos de avaliação envolvem todo o processo de ensino-aprendizagem da intervenção pedagógica, sendo considerado o esforço, a participação, a motivação e as estratégias de resolução do aluno, além dos acertos dos testes, questionários e atividades propostas. A avaliação é processual, abre a possibilidade do diálogo, reforça e direciona, de forma construtiva, o educando na busca do conhecimento a fim de obter uma aprendizagem significativa.

\section{Resultados e discussões}

Para Para atender ao objetivo geral de apresentar uma proposta de intervenção pedagógica com a metodologia de aprendizagem baseada em equipes (TBL) para o ensino/aprendizagem da disciplina matemática financeira do curso técnico de administração integrado ao ensino médio, foi realizada uma pesquisa teórica do tipo exploratória com análise da prática dessa metodologia na literatura produzida sobre o assunto até então e proposta uma estratégia de ensino na disciplina Matemática Financeira com o conteúdo de Séries Uniformes de Pagamentos para os alunos do curso técnico integrado ao ensino médio.

A prática pedagógica, proposta nesta pesquisa, visa a proporcionar o conhecimento e contextualização dos conteúdos teóricos de séries uniformes de pagamentos com o uso do método TBL. O estudo prévio do conteúdo é realizado fora da sala de aula com o acesso à vídeo-aulas, textos e exercícios para resolução com planilhas eletrônicas. Em sala de aula, a verificação da aprendizagem é realizada com a aplicação de um teste individual e, posteriormente, os grupos promovem uma discussão colaborativa em equipe com a finalidade de chegar a um consenso sobre o teste aplicado. Nesta etapa do método, como forma de consolidar todo o conteúdo abordado de forma lúdica e atrativa, é proposto um jogo de tabuleiro de perguntas e respostas entre as equipes com pontuações para avançar nas casas do tabuleiro, se a resposta estiver correta, e recuar caso contrário. Por fim, a aplicação dos conceitos pelo método TBL é realizada fora da sala de aula com a resolução de questões, simulando contextos reais e a criação de um mural interativo, e em sala de aula com a resolução de uma situação prática que possui características e complexidade semelhantes às dificuldades encontradas por um técnico em 
Administração na rotina de suas atividades profissionais. A seguir algumas questões dos testes individuais e coletivos:

1) Diversas linhas de financiamento de energia solar são oferecidas, hoje, no Brasil através de bancos públicos e privados, tornando a aquisição dos sistemas fotovoltaicos mais acessível para boa parte da população. Uma pessoa que gasta em média $700 \mathrm{kWh} /$ mês de energia possui uma conta mensal média de $\mathrm{R} \$ 553,00$ por mês. Retirado o custo de disponibilidade (tarifa mínima de energia elétrica) para o padrão trifásico que é de $100 \mathrm{kWh}$ (equivalente a $\mathrm{R} \$ \mathbf{7 9}, 00$ ), podemos considerar que, idealmente essa pessoa buscará gerar 600kWh/mês de energia solar, ou seja uma economia mensal de $\mathrm{R} \$ 474,00$. Tendo encontrado uma linha de financiamento com juros de $1,0 \%$ ao mês e que a fizesse manter a mesma despesa mensal ( $R \$ 79,00$ da energia elétrica $+R \$ 474,00$ do financiamento) com energia em 10 anos (120 parcelas), essa pessoa adquiriu o sistema fotovoltaico que custou hoje o equivalente a $\mathrm{R} \$ 35.000,00$. Contudo, foi necessário o pagamento de uma entrada para quitar todo o custo, dessa forma, qual foi o valor da entrada?

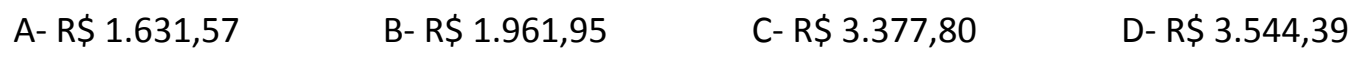

2) Em um financiamento mensal, a notação 1+4 é muito comum. Ela indica que

A- O financiamento tem um período de carência para iniciar os pagamentos e depois mais 4 prestações iguais.

B- O financiamento tem 5 prestações iguais com início dos pagamentos em 1 mês.

C- O financiamento tem uma prestação em 1 mês e outra prestação em 4 meses.

D- O financiamento tem uma prestação na data atual e depois mais 4 prestações iguais.

3) Uma empresa pretende financiar um equipamento em 48 prestações mensais, iguais e postecipadas de $\mathrm{R} \$$ 630,00 . Considerando que a vida útil desse equipamento é de 3 anos, que ele trará retornos mensais ao final do primeiro mês de uso e que ele será inutilizado após esse prazo, não possuindo nenhum valor residual, quanto o equipamento deverá retornar no mínimo, para que a compra seja viável, se a taxa de juros compostos for de $1,5 \%$ ? Desconsidere qualquer outro custo para a operacionalização do equipamento.
A- $R \$ 595,75$
B- $R \$ 775,35$
$C-R \$ 840,00$
$D-R \$ 1093,25$

4) Para comprar um produto que custa $\mathrm{R} \$ 4.916,15$ à vista, um cliente só consegue dispor do seu orçamento mensalmente $R \$ 250,00$. Sabendo que a taxa de financiamentos praticada é de $4 \%$ ao mês e que o cliente vai começar a pagar as prestações no momento da compra, determine em quantas parcelas será financiado o produto?
A- 38
B- 36
C- 32
D- 21

5) Sobre as operações que envolvem determinada quantidade de recebimentos ou pagamentos parcelados, a alternativa que apresenta operações caracterizadas pela condição de vencimento da primeira parcela ocorrer em um período superior ao primeiro período subsequente ao da compra é

A- Operações com carência postecipada.

B- Operações antecipadas.

C- Operações postecipadas.

D- Operações com perpetuidade. 
6) Um servidor com um salário líquido de $R \$ 6.500,00$ já possui $10 \%$ do seu salário comprometido em um empréstimo consignado e agora está pensando em fazer outro. Sabendo que o valor máximo de margem consignável corresponde a $30 \%$ do salário líquido do servidor, qual deve ser o valor da entrada que esse servidor deve pagar para fazer um financiamento de $R \$ 15.000,00$ em 12 parcelas à taxa de 1,5\% ao mês no máximo do crédito consignado?
$A-R \$ 6.269,24$
B- $R \$ 1.664,91$
C- $R \$ 820,84$
$D-R \$ 607,55$

7) Uma agência de automóveis considera a taxa de juros de $30 \%$ a.a capitalizada mensalmente e estabelece o seguinte plano de financiamento para carros com valor de $\mathrm{R} \$ 150.000,00$ : Uma entrada de $\mathrm{R} \$ 50.000,00,1$ prestação anual de $R \$ 30.000,00$, e mais 24 parcelas mensais. Determine qual será o valor da prestação mensal?
$A-R \$ 3.818,44$
B- $R \$ 3.913,90$
$C-R \$ 4.238,10$
$D-R \$ 4.344,05$

8) Uma pessoa fez uma aplicação mensalmente durante 15 meses da seguinte forma: $\mathrm{R} \$ 1.000,00$ durante os primeiros 6 meses, $R \$ 3.000,00$ nos 5 meses seguintes, e $R \$ 4.000,00$ nos últimos 4 meses. Considerando que as aplicações são feitas ao fim de cada mês, calcule o montante acumulado ao fim dos quinze meses, considerando uma taxa de juros compostos de $2 \%$ a.m.
A- $R \$ 40.924,28$
B- $\mathrm{R} \$ 38.406,67$
$C-R \$ 35.944,71$
$D-R \$ 33.202,63$

9) A compra de um caminhão foi financiada em 120 prestações de $R \$ 2.450,00$ à taxa de 1,5\% am, com carência de 4 meses para o início dos pagamentos. Se, além do financiamento, foi paga uma entrada de $\mathrm{R} \$ 20.000,00$, qual seria o valor à vista do caminhão?
$A-R \$ 150.031,58$
B- $R \$ 151.982,05$
$C-R \$ 158.010,78$
$D-R \$ 183.333,33$

10) João estava desempregado e há 12 meses não paga o financiamento mensal de $R \$ 455,50$ do carro que comprou. As parcelas em atraso vêm recebendo juros de 1,1\% ao mês, com isso, devido à portabilidade de crédito, João optou por transferir sua dívida atualizada para outra instituição financeira que trabalha com juros de 1\% ao mês. Sabendo que João parcelou sua dívida acumulada em 1+12 prestações no novo banco, qual será o novo valor das prestações mensais que terá que pagar para quitar seu financiamento?
A- $R \$ 479,23$
B- $R \$ 478,76$
$C-R \$ 474,02$
$D-R \$ 446,02$

As pesquisas, realizadas com aplicação da metodologia aprendizagem baseada em equipe, destacam a eficiência do método na construção do conhecimento. Os resultados encontrados no estudo de Barbiero et al (2017) indicam que a maioria dos respondentes relatou que o método TBL permite uma aprendizagem significativa do conteúdo (71,31\%) e aumenta o interesse para estudar o conteúdo abordado (62,29\%). Resultados semelhantes foram encontrados por Masocattoet al. (2019), onde $91 \%$ dos estudantes relata um impacto positivo em seus estudos e $72 \%$ indica uma melhor compreensão do conteúdo estudado com a metodologia aprendizagem baseada em equipe. Os resultados de Portes (2016) e Camargo et al. (2017) também confirmam que a estratégia educacional é mais eficaz quando envolve o trabalho em equipe. Portes (2016) evidencia o aumento de acertos nas tarefas realizadas em equipe $(78,23 \%$ de acertos) comparado àquelas realizadas individualmente $(64,63 \%)$, assim como Camargo et al. (2017) que revela um aumento nas médias das pontuações das atividades das equipes (7,8 pontos) em relação às médias individuais $(5,5)$.Bollela et al. (2014) destaca que a eficácia do método TBL como estratégia educacional está baseada no estímulo dos estudantes em alcançar um grau de coesão. Dessa forma, uma boa interação entre os membros, resulta em maior aprendizado e motivação para enfrentar os desafios propostos nas atividades. 
Além de melhorar o desempenho dos alunos e promover uma aprendizagem significativa com a aplicação contextualizada dos conceitos, o método TBL desenvolve habilidades de comunicação, argumentação e convencimento, conforme observado no estudo de Oliveira et al. (2018). Os autores destacaram ainda que ao exercitarem essas habilidades, os estudantes melhoraram sua interação com os colegas e foram capazes de desenvolver um trabalho colaborativo em equipe.

É importante destacar que a escolha dessa temática com aplicação na disciplina Matemática Financeira para os alunos do 20 ano do curso técnico de administração integrado ao ensino médio do Instituto Federal do Espírito Santo - campus Colatina se deu porque, a autora principal deste artigo atua como professora da área de administração, há mais de 13 anos leciona essa disciplina, tanto no nível médio como no superior. Contudo, temos encontrado várias dificuldades na relação entre o ensino e a aprendizagem dos alunos, seja por falta de conhecimento da matemática básica, realidade de muitos estudantes, ou mesmo pela ausência de metodologias que motivem e despertem o engajamento do aluno no aprendizado.

Ademais, poucos estudos têm abordado o método de aprendizagem baseada em equipe como estratégia educacional no nível técnico da área de administração. Diante de uma sociedade pautada no conhecimento e na informação e em constante desenvolvimento tecnológico, o mercado tem exigido cada vez mais dos profissionais dessa área e a aplicação de metodologias ativas, como o TBL, no processo de ensino-aprendizagem, pode-se estimular o desenvolvimento de competências fundamentais como pró-atividade, autonomia, tomada de decisão, trabalho em equipe, entre outras, contribuindo para formar profissionais ativos, críticos e reflexivos

Os resultados encontrados por Oliveira et al. (2019) demonstram que a metodologia aprendizagem baseada em equipe promove mudanças positivas nas crenças dos estudantes sobre suas capacidades em aprender. Dos 27 alunos respondentes, 26 obtiveram um aumento na percepção de autoeficácia em aprender o conteúdo abordado.

O método TBL valoriza a responsabilidade individual dos estudantes e motiva para os estudos por ser uma aplicação contextualizada dos conceitos, além de considerar as experiências prévias dos estudantes na busca da aprendizagem significativa (BOLLELA et al., 2014). A participação ativa no processo de ensino-aprendizagem baseado no diálogo e na interação do trabalho efetivo e colaborativo em equipe, além de motivar o aluno a construir o conhecimento, aprimora a comunicação interpessoal e o raciocínio crítico para tomada de decisões (KRUG et al., 2016)

A aplicação desse método na disciplina matemática financeira do curso técnico em administração pode contribuir para a efetiva aprendizagem significativa, o que tem ainda especial relevância social na formação dos alunos, pois esta disciplina está intimamente relacionada a contextos reais, além de possibilitar a discussão sobre conceitos relativos à educação financeira. O estudo desse tema pode contribuir para formar cidadãos conscientes quanto à aplicação dos seus rendimentos, o que proporciona uma gestão mais eficiente das suas finanças pessoais, modificando seu comportamento como consumidor.

De acordo com Pinto et al., (2012, p.78),

Promover a aprendizagem significativa, exige, em primeiro lugar, uma metodologia de ensino que seja capaz de envolver o aluno enquanto protagonista de sua aprendizagem, desenvolvendo ainda o senso crítico diante do que é aprendido, bem como competências para relacionar esses conhecimentos ao mundo real. 
O conteúdo de Séries Uniformes de Pagamentos, abordado na disciplina de matemática financeira, está fortemente relacionado a educação financeira e ao cotidiano das pessoas em concordancia com Pinto et al. , (2012, p.78). Infelizmente, a educação financeira não é uma realidade no currículo escolar de muitas instituições de ensino brasileiras, apesar de sua importância na formação dos educandos. Contudo, a aprendizagem significativa dos conteúdos de matemática financeira pode contribuir para decisões relacionadas ao planejamento financeiro que são essenciais a um melhor gerenciamento financeiro pessoal, como a decisão de comprar à vista ou a prazo e a escolha entre os diferentes tipos de investimentos e financiamentos disponíveis.

Para Assaf Neto, (2019), a matemática financeira, em essência, estuda o valor do dinheiro no tempo e tem o objetivo de analisar e comparar os fluxos de caixa, com entrada e saída de dinheiro, em diversos momentos. Dessa forma, adiar um recebimento, por exemplo, envolve um sacrifício e por isso deve-se ter uma recompensa que é o juro (Assaf Neto, 2019).

As Séries Uniformes de Pagamentos envolvem situações financeiras com mais de um pagamento, ou recebimento, de mesmo valor monetário, ocorrendo em intervalos de tempo iguais (Branco, 2015). Pode ser ilustrada conforme Figura 2, onde 'PMT' significa pagamento, o valor constante das prestações, e ' $n$ ' refere-se a quantidade de prestações.

Figura 2

Fluxo de caixa de uma série uniforme de pagamentos

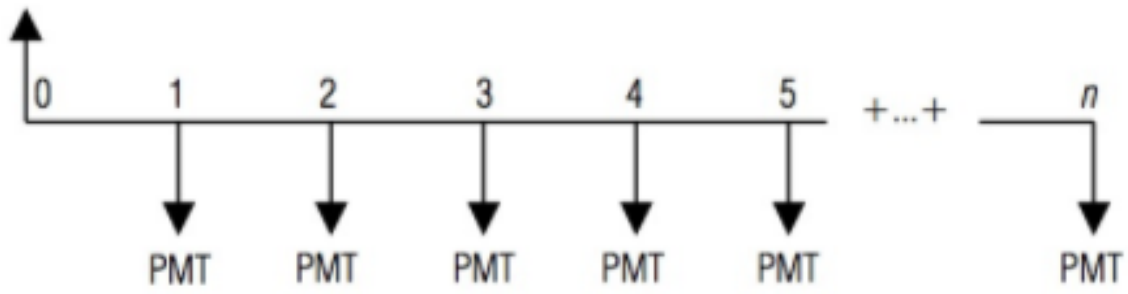

Fonte: Branco (2015)

Quanto ao período de ocorrência do primeiro pagamento, as séries uniformes podem ser classificadas em postecipadas, antecipadas ou diferidas. As séries são postecipadas quando o primeiro pagamento ocorre ao final de um período, ou seja, se as prestações uniformes são mensais por exemplo, o primeiro pagamento deverá ocorrer daqui um mês. Já nas séries antecipadas, o primeiro pagamento ocorre no momento zero, ou seja, o fluxo de pagamentos/recebimentos constantes ocorre no início do período, como acontece em parcelamentos com entrada que possui o mesmo valor do restante das prestações. As séries classificadas como diferidas, possuem um prazo superior a um período para o início dos pagamentos/recebimentos, sendo assim, o primeiro pagamento ocorrerá após um período de carência.

Algebricamente, podemos resolver as questões envolvendo situações de séries uniformes de pagamentos/recebimentos com o uso de tabelas financeiras ou através das fórmulas, sintetizadas no Quadro 1, com o uso de uma calculadora científica, onde $P M T$ refere-se ao valor da prestação constante, $n$ significa a quantidade de parcelas da série de pagamentos/recebimentos, i é a taxa de juros compostos aplicada, VP é o valor presente da dívida ou do investimento, VF significa valor futuro, valor acumulado após a série de pagamentos periódicos. 
Quadro 1

Fórmulas das séries uniformes de pagamentos

\begin{tabular}{|c|c|c|}
\hline & Valor Presente & Valor Futuro \\
\hline Postecipada & $V P=P M T\left[\frac{1-(1+i)^{-n}}{i}\right]$ & $V F=P M T\left[\frac{(1+i)^{n}+1}{i}\right]$ \\
\hline Antecipada & $V P=P M T\left[\frac{1-(1+i)^{-n}}{i}\right] \cdot(1+i)$ & $V F=P M T\left[\frac{(1+i)^{n}+1}{i}\right] \cdot(1+i)$ \\
\hline Diferida & $V P=P M T\left[\frac{1-(1+i)^{-n}}{i \cdot(1+i)^{c-1}}\right]$ & \\
\hline
\end{tabular}

Fonte: Branco (2015). Adaptado pelos autores

As calculadoras financeiras, como a HP-12C, e as planilhas eletrônicas, como o Excel ${ }^{\bullet}$, possuem funções financeiras predefinidas e são importantes ferramentas para resolver de forma simplificada, as situações que envolvem séries uniformes de pagamentos/recebimentos.

No Excel $^{\oplus}$ cada valor de um elemento (PMT, i, n, VP ou VF) deve estar em uma célula diferente e a função financeira a ser inserida depende do que se espera encontrar. Por exemplo, se a questão de série uniforme estiver procurando a taxa de juros de um financiamento, a função a ser inserida será TAXA. Se estiver buscando o valor presente de uma série de pagamentos/recebimentos, a função será VP. Se o que se quer encontrar na questão é a quantidade de prestações, a função a ser inserida será NPER. Caso a questão esteja procurando o valor das prestações periódicas, a função financeira será PGTO. E, se estiver buscando o valor futuro de uma série de pagamentos/recebimentos, a função será VF.

A Figura 3 traz um exemplo da aplicação de uma situação de série uniforme de pagamentos no Excel ${ }^{\bullet}$, onde se espera encontrar o valor da prestação de uma série de pagamentos iguais, cujo valor presente financiado é de $\mathrm{R} \$ 10.000,00$, parcelado em 24 prestações mensais numa taxa de juros compostos de $1 \%$ ao mês. Cada elemento da questão (VP, i, n) é inserido nos argumentos da função PGTO. Além dos elementos, deve-se especificar o tipo de série que a questão se refere, com 0 para série postecipada e 1 para antecipada. Caso a questão não informe quando será o início dos pagamentos, deve-se considerar uma série postecipada.

Figura 3

Argumentos da função PGTO no Excel ${ }^{\oplus}$

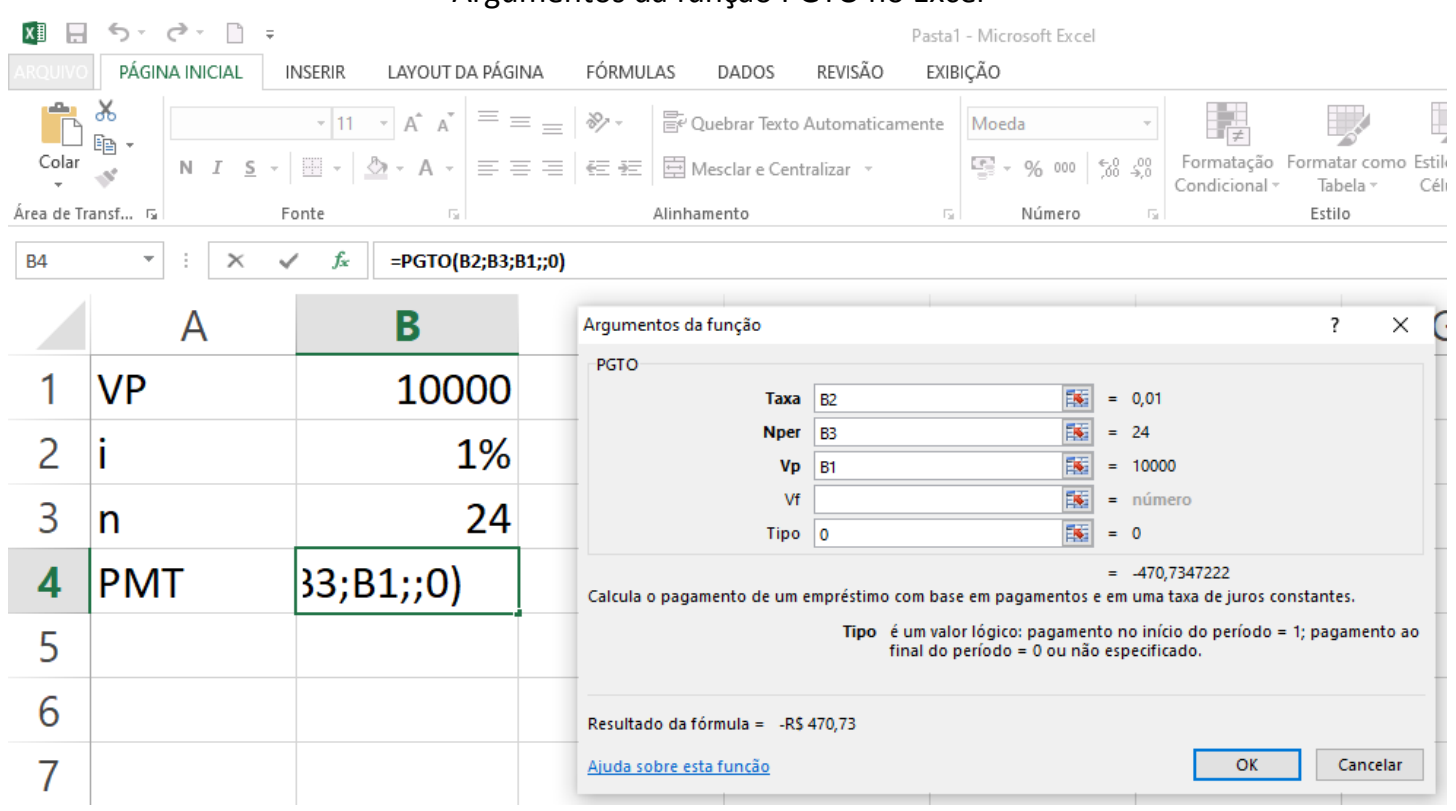

Fonte: Os autores (2020) 
As séries diferidas, com início dos pagamentos após um período de carência, podem ser resolvidas no Excel ${ }^{\circ} \operatorname{com}^{\circ}$ o cálculo do valor futuro do período de carência total, sendo, posteriormente, associado ao valor presente de uma série antecipada.

O conteúdo de séries uniformes de pagamentos está relacionado a contextos reais de situações financeiras dos indivíduos. É aplicado em diversos casos, como o financiamento de imóveis e veículos, parcelamento na compra de produtos, parcelamento de dívidas em cartões de crédito, aplicações em investimentos de renda fixa com recebimento de juros periódicos, portabilidade de dívidas com empréstimos, entre outros. Entender como os juros se comportam ao longo do tempo é importante para formar cidadãos conscientes quanto à aplicação dos seus recursos financeiros e a prática pedagógica com o método TBL pode contribuir para a efetiva aprendizagem significativa desse conteúdo.

\section{Conclusões}

O presente artigo trouxe uma análise da prática da metodologia aprendizagem baseada em equipes (TBL) e propôs uma intervenção pedagógica na disciplina Matemática Financeira com o conteúdo de Séries Uniformes de Pagamentos para os alunos do curso técnico integrado ao ensino médio.

A aplicação do método TBL, como estratégia pedagógica, tem crescido no ambiente educacional e se destacado como uma metodologia eficaz na construção do conhecimento. Os resultados das pesquisas decorrentes da aplicação de práticas pedagógicas com a aprendizagem baseada em equipe, destacam a postura ativa, a motivação e a interação dos estudantes entre os pares, com o desenvolvimento do trabalho colaborativo.

Apesar de a intervenção pedagógica proposta nessa pesquisa não ter sido aplicada, devido à pandemia da COVID19, acredita-se que a aprendizagem baseada em equipes se mostra como uma estratégia educacional apropriada a disciplina matemática financeira no curso técnico de administração integrado ao ensino médio. 0 método promove uma visão crítica e reflexiva, relacionando conteúdos teóricos a contextos reais, estimulando o aluno a desenvolver competências fundamentais a atividade profissional e contribuindo para a formação do educando bem como para sua efetiva aprendizagem significativa.

\section{Referências bibliográficas}

Anjos, R., Rodriguez, J., Fujiki, R., \& Bernardo, M. (2016). Aprendizagem baseada em equipe: uma estratégia de ensino aplicada na área da imaginologia. Revista Da Faculdade de Ciências Médicas de Sorocaba, 18, 90.

Assaf Neto, A. (2019). Matemática financeira e suas aplicações (14th ed.). Atlas.

Barbiero, A. J. C., Honorato, A. A., Vieira, C. F., Silva, G. T. G. da, Ferreira, I. M., Barreiros, L. L., Silva, L. F. L. e, Silva, M. L. F. L. e, Segheto, W., \& Fontes, L. B. A. (2017). Percepção dos acadêmicos de medicina sobre a metodologia de aprendizagem baseada em equipes na disciplina de farmacologia. Revista Científica FAGOC-Saúde, 2(2), 43-49.

Bollela, V. R., Senger, M. H., Tourinho, F. S. V., \& Amaral, E. (2014). Aprendizagem baseada em equipes: da teoria à prática. Medicina (Ribeirao Preto. Online), 47(3), 293. https://doi.org/10.11606/issn.21767262.v47i3p293-300

Branco, A. C. C. (2015). Matemática financeira aplicada: Método Algébrico, HP-12C e Microsoft Excel ${ }^{\circledR}$ (Cengage Learning Brasil (ed.); 4th ed.). 
Camargo, F., Pitaguari, A., \& Dalberto, D. M. (2017). O Uso do Team-Based Learning como Estratégia de Avaliação Formativa no Curso de Administração da Uniamérica. CAMARGO, Fausto; PITAGUARI, Antonio; DALBERTO, Dirce Maria, 11(21), 77-89.

Espinosa, T., Araujo, I. S., \& Veit, E. A. (2019). Crenças de autoeficácia em aprender física e trabalhar colaborativamente: um estudo de caso com o método team-based learning em uma disciplina de física básica. Revista Brasileira de Ensino de Ciência e Tecnologia, 12(1).

https://doi.org/10.3895/rbect.v12n1.6020

GIACOMELLI, S. C. P. (2020). O uso da metodologia TEAM-BASED LEARNING (TBL) aliada à tecnologia: percepções sobre a aprendizagem de contabilidade básica no Curso Técnico em Administração. Universidade do Oeste Paulista UNOESTE.

GIL, A. C. (2002). Como elaborar projetos de pesquisa (4th ed.). Atlas.

Huang, C.-K., \& Lin, C.-Y. (2017). Flipping Business Education: Transformative Use of Team-Based Learning in Human Resource Management Classrooms. Journal of Educational Technology \& Society, 20(1), 323-336.

Kibble, J. D., Bellew, C., Asmar, A., \& Barkley, L. (2016). Team-based learning in large enrollment classes. Advances in Physiology Education, 40(4), 435-442. https://doi.org/10.1152/advan.00095.2016

Krug, R. de R., Vieira, M. S. M., Maciel, M. V. de A. e, Erdmann, T. R., Vieira, F. C. de F., Koch, M. C., \& Grosseman, S. (2016). O "Bê-Á-Bá" da Aprendizagem Baseada em Equipe. Revista Brasileira de Educação Médica, 40(4), 602-610. https://doi.org/10.1590/1981-52712015v40n4e00452015

Marconi, M. de A., \& Lakatos, E. M. (2010). Fundamentos De Metodologia Científica (atlas).

Masocatto, N. O., Couto, W. J., Matta, T. S. da, \& Porfirio, G. J. M. (2019). Percepção de Alunos de Curso de Graduação em Medicina sobre o Team-Based Learning (TBL). Revista Brasileira de Educação Médica, 43(3), 111-114. https://doi.org/10.1590/1981-52712015v43n3rb20180231

Oliveira, B. L. C. A. de, Lima, S. F., Rodrigues, L. dos S., \& Pereira Júnior, G. A. (2018). Team-Based Learning como Forma de Aprendizagem Colaborativa e Sala de Aula Invertida com Centralidade nos Estudantes no Processo Ensino-Aprendizagem. Revista Brasileira de Educação Médica, 42(4), 86-95. https://doi.org/10.1590/1981-52712015v42n4rb20180050

Oliveira, T. E. de. (2016). Aprendizagem de física, trabalho colaborativo e crenças de autoeficácia: um estudo de caso com o método Team-Based Learning em uma disciplina introdutória de eletromagnetismo. [Universidade Federal do Rio Grande do Sul (UFRGS)]. http://hdl.handle.net/10183/135013

Oliveira, T. E. de, Araujo, I. S., \& Veit, E. A. (2016). Aprendizagem Baseada em Equipes (Team-Based Learning): um método ativo para o Ensino de Física. Caderno Brasileiro de Ensino de Física, 33(3), 962. https://doi.org/10.5007/2175-7941.2016v33n3p962

Paiva, M. R. F., Parente, J. R. F., Brandão, I. R., \& Queiroz, A. H. B. (2016). Metodologias ativas de ensinoaprendizagem: revisão integrativa. SANARE-Revista de Políticas Públicas, 15(2), 145-153.

PINTO, A. S. S., BUENO, M. R. ., SILVA, M. A. F. ., SELLMANN, M. ., \& KOEHLER, S. M. F. (2012). METODOLOGIAS ATIVAS DE ENSINOAPRENDIZAGEM: REVISÃO INTEGRATIVA. Revista de Pesquisa Científica - Janus - Fatea, 9(15), 75-87.

PORTES, S. da S. (2016). Intervenção pedagógica mediada com metodologia ativa Team Based Learning na educação profissional: uma experiência no Instituto Federal de Brasília. Outras Palavras, 12(2), 78. 
RICHARDSON, R. J. (1999). Pesquisa social: métodos e técnicas. Atlas.

Saldivar, K. M. (2015). Team-Based Learning: A Model for Democratic and Culturally Competent 21st Century Public Administrators. Journal of Public Affairs Education, 21(2), 143-164.

https://doi.org/10.1080/15236803.2015.12001825

Smart, K. L., Berry, R., Kumar, A., Kumar, P., \& Scott, J. P. (2015). Developing a Preference for Collaboration Using Team-Based Learning. Journal on Excellence in CollegeTeaching, 26(3), 165-189.

Uzinski, J. C., Oliveira, L. F. S. de, Silva, \& Almeida, L. Da. (2019). Ensino de matemática financeira no curso de licenciatura em matemática e bacharelado em ciências contábeis: reflexões dos impactos no processo de ensino/aprendizagem e/ou no mercado de trabalho. In B. R. de Oliveira, A. Mario Zuffo, \& J. González Aguilera (Eds.), Caminhos da Matemática História, Educação e Aplicações (1st ed., pp. 51-60). Pantanal Editora. https://doi.org/10.46420/9786581460020

VILELA, R. Q. B., BANDEIRA, D. M. A., \& SILVA, M. A. (2017). Aprendizagem Baseada em Equipe. Revista Portal: Saúde e Sociedade, 2(1), 371-379. https://doi.org/10.28998/rpss.v2i1.2707

Zgheib, N. K., Dimassi, Z., Bou Akl, I., Badr, K. F., \& Sabra, R. (2016). The long-term impact of team-based learning on medical students' team performance scores and on their peer evaluation scores. Medical Teacher, 38(10), 1017-1024. https://doi.org/10.3109/0142159X.2016.1147537

Esta obra está bajo una Licencia Creative Commons Attribución-NoCommercial 4.0 International

\section{$(\mathrm{Cc})$ EY-NO}

